УДК 621.372.037(075.8)

DOI https://doi.org/10.32838/2663-5941/2020.4/07

Ушкаренко А.О.

Национальный университет кораблестроения имени адмирала Макарова

\title{
ИСПОЛЬЗОВАНИЕ МНОГОУРОВНЕВОЙ ДЕКОМПОЗИЦИИ ПРИ РАЗРАБОТКЕ ГРАФОАНАЛИТИЧЕСКИХ МОДЕЛЕЙ ПОДСИСТЕМ ЦИФРОВОЙ ОБРАБОТКИ СИГНАЛОВ
}

У статті розглядається вдосконалений метод опису структур изифрових фільтрів, який відрізняється підвищеним інформаційним змістом отриманих моделей систем ичфрової обробки сигналів. Використання запропонованого підходу дає змогу виконати синтез графоаналітичних моделей систем цифрової обробки сигналів та провести ії аналіз шляхом багаторівневої декомпозииії. Це дає змогу оцінити якість структури системи та елементів, щзо входять до ї̈ складу, з позицій загального системного підходу, і спрощує проведення інтеграційного тестування системи изиррової обробки сигналів за рахунок формального визначення всіх можливих схем взаємодії апаратно-програмних засобів. При цьому всі елементи системи представлені у вигляді функціональних структур, а обробка дискретною системою вхідного відліку розглядається як процес перетворення вхідних аргументів за допомогою иих функиіональних структур. Розроблено правила формального опису елементів систем цифрової обробки сигналів і отримані графоаналітичні моделі базових структур нерекурсивних і рекурсивних иифрових фільтрів, відмінною рисою яких є підвищений інформаційний зміст. Практична цінність отриманих результатів полягає в можсливості використання формального підходу в проиесі вирімення завдання синтезу оптимізованих структур цифрових фільтрів. Запропонований у статті метод використаний при синтезі оптимізованої структури цифрового фільтра для обчислення середнього і діючого значень детермінованого періодичного сигналу при оптимальній швидкодї дискретної системи та інваріантності до частоти дискретизаиії. Час, необхідний для обчислення чергового вихідного значення оптимізованим иифровим фільтром, у 2,5 раза менше ніж час, який необхідний для виконання всіх обчислень при використанні неоптимізованої структури цифрового фільтру. Представлені в статті моделі структур иифрових фільтрів можуть бути використані в радіоелектронних та телекомунікачійних системах різного призначення у вигляді вбудовуваних модулів після їх інтеграчї з об'єктно орієнтованою мовою програмування.

Ключові слова: цифровий фільтр, функиіональна структура, аналітична модель, оптимізація, перетворення аргументів.

Постановка проблемы. Цифровая обработка сигналов имеет большое фундаментальное и прикладное значение в электронике, телекоммуникациях, радиотехнике и смежных отраслях. Современные радиотехнические и телекоммуникационные системы характеризуются большим количеством элементов, множеством связей и значительным объемом обрабатываемой информации. Для разработчика электронной и телекоммуникационной аппаратуры важно глубокое понимание процессов преобразования сигналов, которое выполняет система, для разработки соответствующих алгоритмов.

Все чаще возникает необходимость в разработке более сложных цифровых систем в более короткие сроки. В таких случаях работа на компонентном уровне больше не является эффективной, что приводит к необходимости работать на более высоком уровне абстракции проекта [1, с. 43].
Здесь проектировщик разрабатывает и использует высокоуровневые системные модели, которые затем реализуются схемотехнически с использованием регистровой логики или программно в виде исходного кода на одном из языков высокого уровня. Поэтому актуальной является задача усовершенствования метода описания систем цифровой обработки сигналов и ее отдельных элементов, а также применение алгоритмов цифровой фильтрации для улучшения качества функционирования радиотехнических и телекоммуникационных систем.

Анализ последних исследований и публикаций. Известны несколько эквивалентных форм представления цифровых фильтров. Это представление в форме передаточной функции, разностных уравнений, в виде разложения передаточной функции на простые дроби, в каскадной форме, в пространстве состояний, а также в виде 
$z p$-представления [2, с. 133; 3, с. 27]. При этом информация о структуре системы обработки сигналов или отсутствует, или представлена в неявном виде. Также широко используется описание в виде структурных схем, принципиальных схем, а также системы моделирования Matlab Simulink [4, с. 60; 5, с. 126], SciCos [6, с. 38], Proteus, Multisim и другие, в которых исследуемые системы, а также их элементы, представлены с использованием различных уровней абстрагирования, полученных в результате декомпозиции исходной системы на отдельные подсистемы [7, с. 1335]. Однако основным недостатком такого описания систем является отсутствие информации о структуре ее отдельных элементов, которые рассматриваются как «черный ящик». Глубина описания, уровень детализации определяется назначением системы и используется для построения моделей систем. Изучение особенностей этой информации является предметом анализа систем [8, с. 3295].

К параметрам цифровых фильтров в современных системах цифровой обработки сигналов (ЦОС) предъявляются повышенные требования $[9$, с. $29 ; 10$, с. 116]. Порядки фильтров нередко достигают тысяч и более. Это ведет к увеличению объемов вычислений и резкому росту аппаратных затрат, а в случае программной реализации фильтра - к увеличению затрат процессорного времени на выполнение всех необходимых вычислений. При синтезе цифровых фильтров наибольшие затраты времени и оборудования приходятся на операции умножения [11, с. 128]. Таким образом, задача минимизации времени вычислений и уменьшения аппаратных затрат сводится к уменьшению количества умножений, необходимых для вычисления очередного отфильтрованного отсчета [12, с. 133]. При этом главное внимание в литературе уделяется вопросам параметрической оптимизации цифровых фильтров как при их проектировании на основе аналоговых прототипов, так и при разработке адаптивных фильтров $[13$, с. $122 ; 14$, с. 336$]$. В связи с этим методы описания структур систем ЦОС и процессов преобразования сигналов в них требуют усовершенствования, и как показывает проведенный анализ литературы, имеют значительные резервы для развития.

Предлагаемый в этой работе подход к описанию систем ЦОС расширяет и дополняет методы описания структур цифровых систем управления и логико-динамических процессов преобразования информации, представленные в работах $[15$, c. $279 ; 16$, c. 30$]$.
Постановка задания. Целью статьи является усовершенствование метода описания структур цифровых нерекурсивных и рекурсивных фильтров для построения их графоаналитических моделей, в которых все элементы представлены в виде функциональных структур, выполняющих преобразование входных аргументов, что позволит представить системы ЦОС на различных уровнях декомпозиции и реализовать формальный подход к решению задач их структурной оптимизации.

Для достижения поставленной цели необходимо:

1) проанализировать работу нерекурсивных и рекурсивных фильтров с точки зрения взаимодействия различных функциональных структур, а обработку входного отсчета дискретной системой рассмотреть как процесс преобразования аргументов с помощью этих функциональных структур;

2) разработать правила формального описания элементов систем цифровой обработки сигналов и представить структуры нерекурсивных и рекурсивных цифровых фильтров в виде графоаналитических выражений, отличительной особенностью которых является повышенное информационное содержание;

3) разработать оптимизированную структуру подсистемы цифровой обработки сигналов для вычисления среднего и среднеквадратического значений периодического сигнала, отличительной особенностью которой является независимость количества вычислений для получения выходного отсчета от частоты дискретизации входного сигнала.

Изложение основного материала исследования. Если входной сигнал подсистемы ЦОС рассматривать как аргумент, а выходной сигнал как функцию, то подсистема представляет собой некоторую функциональную структуру. Таким образом, полное описание системы можно представить в виде взаимодействующих функциональных структур, каждая из которых выполняет преобразование входных аргументов. Первый уровень понимания процессов преобразования сигналов реализован для разработчика в виде структурной схемы и принципиальной схемы. Второй уровень понимания реализован в виде вычислительных математических моделей с входными и преобразованными аргументами.

Если простейшую схемную реализацию фильтра нижних частот (ФНЧ) первого порядка на основе одного элемента задержки $Z^{-1} \rightarrow 《 f_{1}\left(\mathrm{Rs} \mathrm{Z}^{-1}\right) »$ представить в виде регистра памяти и сумматора $f_{1}(\Sigma)$, которые записать в виде графоаналитического 
выражения, представленного на рис. 1, а, то это выражение может быть записано в виде аналитического выражения вида:

$$
x(n) \rightarrow f(\text { Action }) \rightarrow y(n),
$$

которое адекватно отображает процесс преобразования аргументов и полностью соответствует понятию функциональной структуры, с помощью которой складываются прямой сигнал «x(n)» и задержанный сигнал (или отсчет) с выхода элемента задержки. Для функциональной структуры регистра памяти «Action» представляет собой задержку на один такт. Тогда, согласно этой формы записи, элемент задержки $Z^{-1}$ будет обозначаться как «f $\left.f_{\mathrm{RS}} \mathrm{Z}^{-1}\right) »$, сумматор обозначается как « $f_{1}(\Sigma) »$, а умножитель - как « $f_{1}\left(\Sigma_{\Sigma}\right) »$, поскольку с математической точки зрения умножение - это многократное сложение. При этом в обозначении функциональной структуры, выполняющей задержку входного отсчета на один такт, используется дополнительная информация, отражающая некоторые особенности реализации этого элемента. В данном случае указано, что в составе элемента задержки имеются RS-триггеры. Этот тип триггеров является базовым для построения триггеров других типов, в частности D-триггеров. B свою очередь, на основе D-триггеров строятся регистры памяти, которые и используются при аппаратной реализации линий задержки в системах ЦОС. Таким образом, становится частично известным внутреннее устройство (теоретическая структура) элементов, из которых состоит система ЦОС. Такие элементы в графоаналитической модели представляют собой «серый ящик».

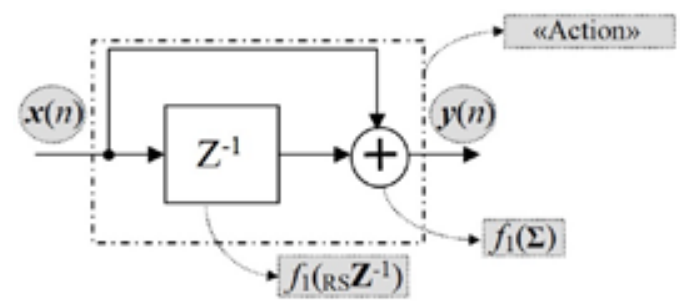

a

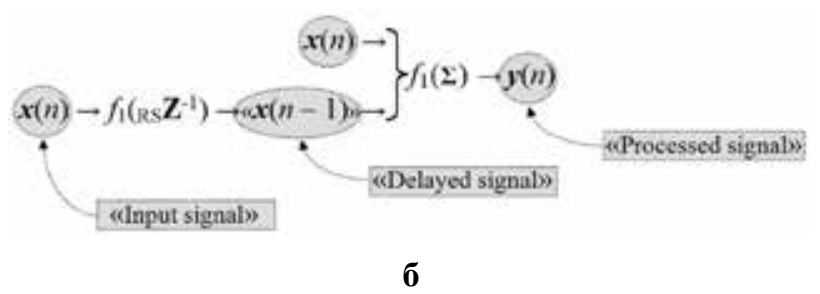

Рис. 1. Структура аппаратной реализации КИХ-фильтра (а) и графоаналитическое выражение, которое описывает функциональную структуру фильтра (б)
Если вернуться к общепринятой математической записи логико-динамического процесса преобразования входного аргумента $x(n)$ графоаналитического выражения (рис. 1, a), то работа такого фильтра нижних частот описывается разностным уравнением вида:

$$
y(n)=x(n)+x(n-1),
$$

При аналитическом описании структур цифровых фильтров символ «\}» математического анализа является «идеальным» символом. Он включает в себя содержание функциональной структуры и может быть уточнен с применением различных индексов и символов, которые широко используются в науке [16, с. 30].

Если структуру фильтра (рис. 1, а) представить в виде аналитического выражения (1), то может быть сформировано аналитическое выражение функциональной структуры фильтра, представленное на рис. 1, б. В этом выражении, в отличие от разностного уравнения (2), исходным является «Input signal», над которым выполняются определенные действия для активизации «Processed signal» $y(n)$.

Вариант построения фильтра ФНЧ/ФВЧ первого порядка с одним полюсом представлен в виде структурной схемы на рис. 2.

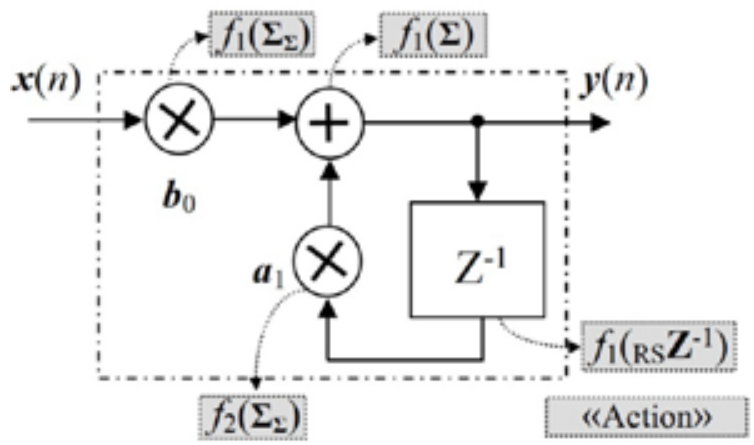

Рис. 2. Структура аппаратной реализации рекурсивного цифрового фильтра

В этой схеме фильтра с бесконечной импульсной характеристикой (БИХ) используется обратная связь, с помощью которой складываются прямой и задержанный сигналы:

$$
y(n)=b_{0} x(n)+a_{1} y(n-1),
$$

$\mathrm{Z}$ - преобразование этого уравнения и передаточная функция фильтра определяются уравнениями:

$$
\begin{gathered}
Y(z)=b_{0} X(z)+a_{1} z^{-1} Y(z), \\
H(z)=\frac{b_{0}}{1-a_{1} z^{-1}} .
\end{gathered}
$$


Для этой схемы фильтра, если $a_{1}>0$ - это ФНЧ, если $a_{1}<0$ - это ФВЧ [9, с. 35]. Если структуру фильтра (рис. 2) записать в виде графоаналитического выражения (1), то вместо алгебраического выражения (3) может быть сформировано графоаналитическое описание функциональной структуры, представленное на рис. 3. В этом выражении, в отличие от разностного уравнения (3), исходным является «Input signal» и «Delayed feedback signal», над которыми выполняются определенные действия для активизации «Processed signal» $y(n)$. Направление передачи данных отображается с помощью стрелки ( $\uparrow)$. Входной информационный поток обозначается стрелкой вниз $(\downarrow)$.

Для рекурсивного фильтра 2 порядка (биквадратный фильтр) может быть сформирована графоаналитическая модель функциональной структуры, представленная на рис. 4.
Из анализа полученной графоаналитической модели можно видеть, что в структуре цифрового фильтра имеется 5 умножителей, 4 сумматора и 2 линии задержки, которые представляют собой регистры памяти. При этом видно, что быстродействие фильтра может быть увеличено за счет параллельного выполнения процессов вычислений. В частности, процедуры умножения, выполняемые функциональными структурами умножителей $f_{2}, f_{3}, f_{4}, f_{5}$, могут быть выполнены параллельно при выполнении алгоритма на многоядерном процессоре, что следует учитывать при программной реализации фильтра. Представление структур фильтров в виде структурных схем или разностных уравнений не позволяет сделать таких выводов. Также могут параллельно выполняться операции суммирования сумматорами $f_{3}$ и $f_{4}$.

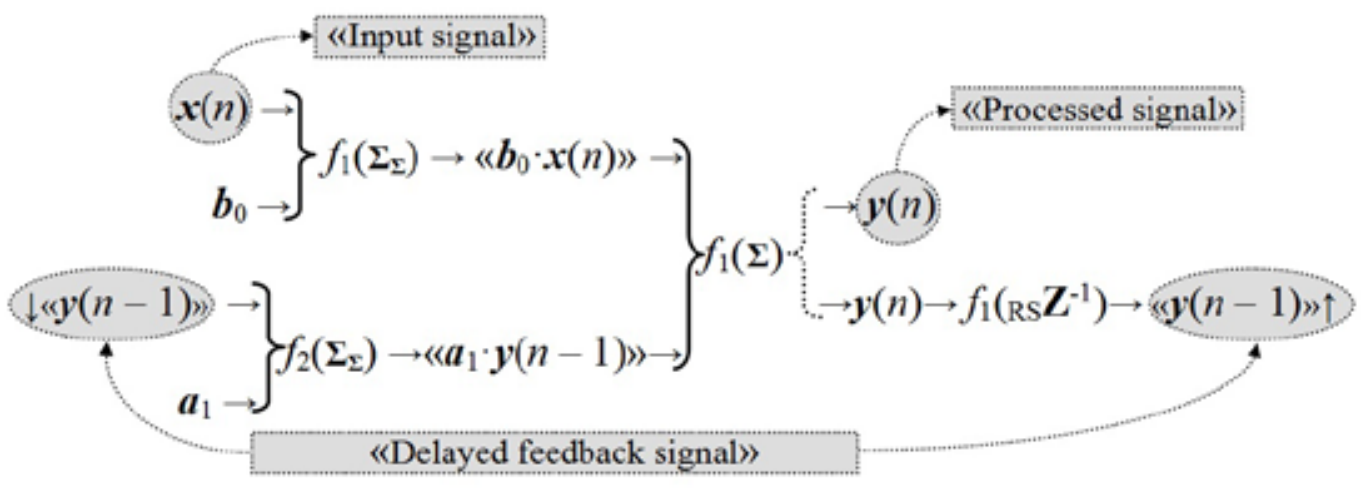

Рис. 3. Графоаналитическое описание функциональной структуры цифрового БИХ-фильтра

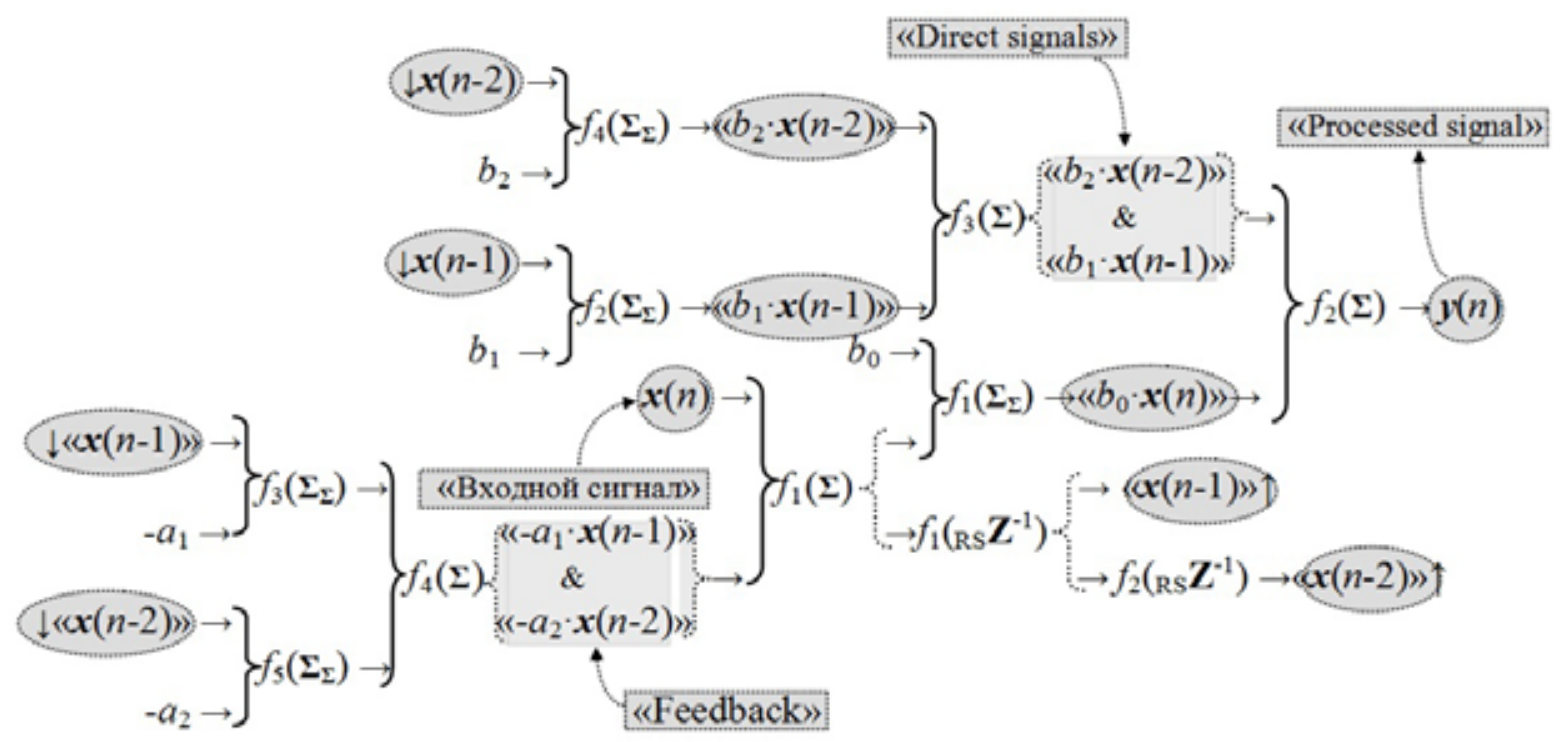

Рис. 4. Графоаналитическое описание биквадратного фильтра 
Рассмотренные графоаналитические модели базовых структур цифровых фильтров являются «строительными блоками» более сложных структур систем ЦОС. Интеграция полученных моделей с объектно-ориентированным языком программирования позволяет их использовать в виде встраиваемых модулей в различных проектах.

Часто задача оптимизации цифровых фильтров состоит в поиске необходимых весовых коэффициентов, при которых характеристики фильтра удовлетворяют некоторому критерию качества $[9$, с. $36 ; 11$, с. $127 ; 17$, с. 100$]$. В таких случаях речь идет о параметрической оптимизации систем ЦОС. Задачи структурной оптимизации могут решаться разработчиками интуитивно, на основе накопленного опыта. При этом получившаяся в результате структура не обязательно будет оптимальной. Предложенный в данной работе подход можно использовать для формального подхода к решению задачи структурной оптимизации систем ЦОС, в частности для синтеза оптимизированной структуры цифрового фильтра для вычисления среднеквадратического значения напряжения. Арифметическое среднее значение переменного сигнала с периодом $T$ рассчитывается по известной математической формуле:

$$
u_{\text {avg }}=\frac{1}{T} \int_{0}^{T}|u(t)| d t,
$$

которую можно записать в виде графоаналитической модели, представленной на рис. 5.

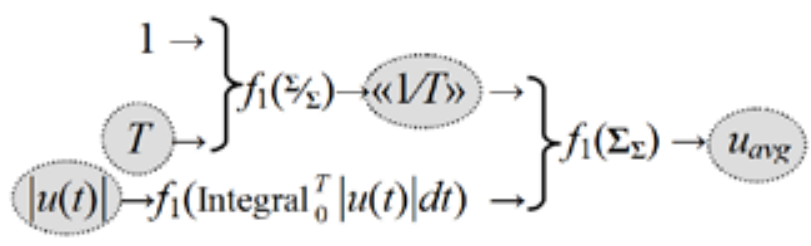

Рис. 5. Графоаналитическая модель процесса интегрирования периодического сигнала

Непрерывный входной сигнал может быть преобразован в последовательность дискретных значений, если с помощью элемента выборкихранения через равные интервалы времени $t_{n}=n \cdot T_{s}$ брать значения входного сигнала. Здесь $T_{s}$ - период дискретизации. Тогда выражение для расчета среднего значения может быть представлено в дискретной форме в виде математической формулы:

$$
u_{\text {avg }}(t)=\frac{1}{N} \sum_{n=0}^{N-1}\left|u\left(t-n T_{s}\right)\right|
$$

где

$$
N=\frac{T}{2 \cdot T_{s}}
$$

Выражение (4) можно записать в виде графоаналитической модели, представленной на рис. 6 .

Далее вводится в рассмотрение обозначения $Y=u_{\text {avg }}, X=|u|$. Переписав уравнение (4), внеся константу $h=1 / N$ под знак суммы и переходя к дискретному времени, получаем следующее выражение:

$$
Y\left(k T_{s}\right)=\sum_{n=0}^{N-1} h \cdot X\left[(k-n) T_{s}\right],
$$

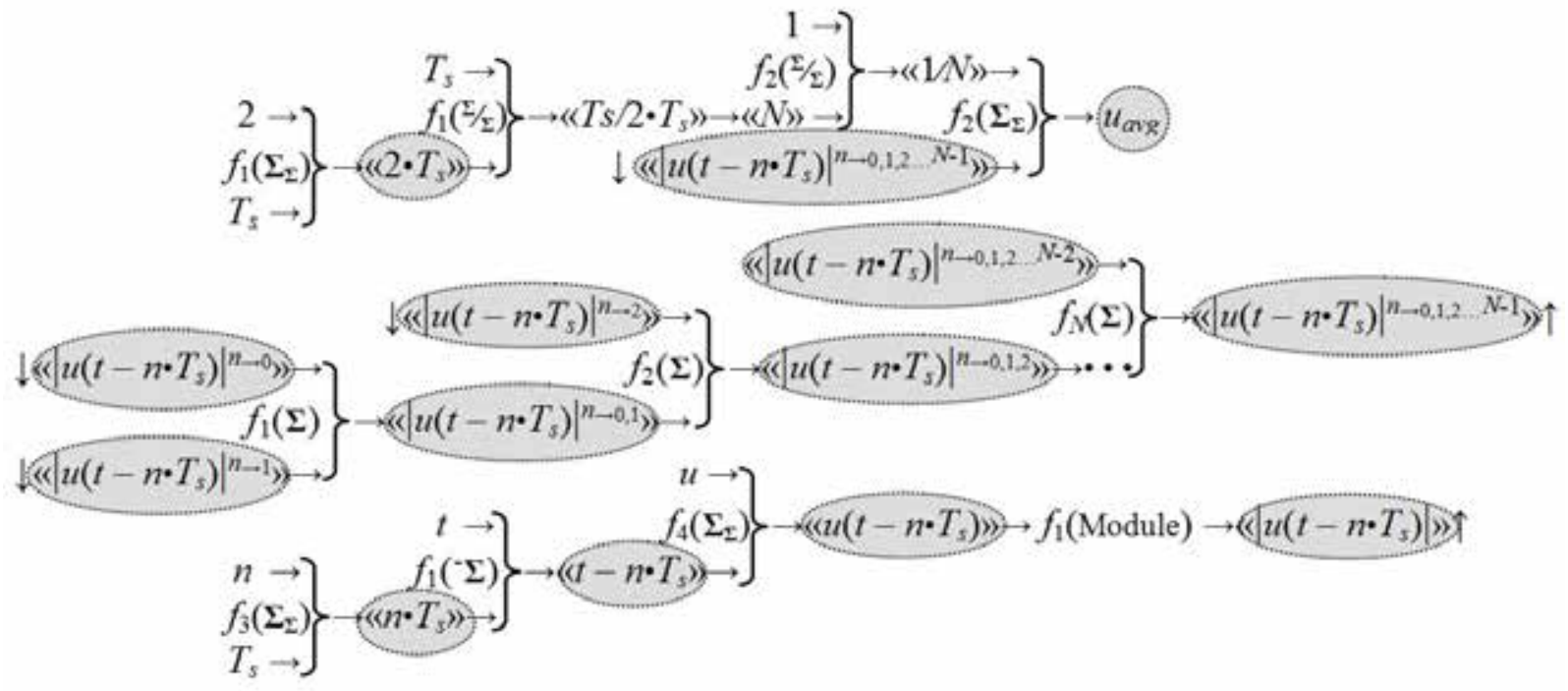

Рис. 6. Графоаналитическая модель системы для расчета среднего значения периодического сигнала 
В результате для активизации конечного результирующего аргумента $Y\left(k T_{s}\right)$ выражения (5) необходимо выполнить преобразование входных аргументов в соответствии с графоаналитической моделью, представленной на рис. 7.

Представленная графоаналитическая модель описывает структуру линейного фильтра с импульсной характеристикой конечной длительности $N-1$.

На рис. 8 представлена графоаналитическая модель оптимизированной структуры цифрового фильтра. Для вычисления очередного выходного отсчёта оптимизированного цифрового фильтра необходимо выполнить 3 операции сложения и одну операцию умножения, против $N$ операций сложения и $N$ операций умножения в исходной структуре. Платой за снижение количества арифметических операций является увеличение длины цифровой линии задержки на один элемент памяти и выделение дополнительного элемента памяти для хранения промежуточного результата. Введя дополнительные функциональные блоки $A^{2}$ и $\sqrt{A}$, оптимизированную структуру цифрового фильтра для расчета среднего значения периодического сигнала с равными весовыми коэффициентами можно использовать для вычисления действующего (среднеквадратического) значения входного сигнала.

Для проверки эффективности полученной структуры цифрового фильтра проводился эксперимент с использованием микроконтроллера ATMega128, работающего от внешнего генератора с тактовой частотой 8 МГц. На вход фильтров была подана ступенчатая функция (функция Хэвисайда) для определения значения интервала времени, необходимого цифровым фильтрам для выполнения всех вычислений и формирования выходного значения. Эксперимент проводился для разных значений порядков цифровых фильтров $16,32,64,128,256,512$ и 1000. В качестве ком-

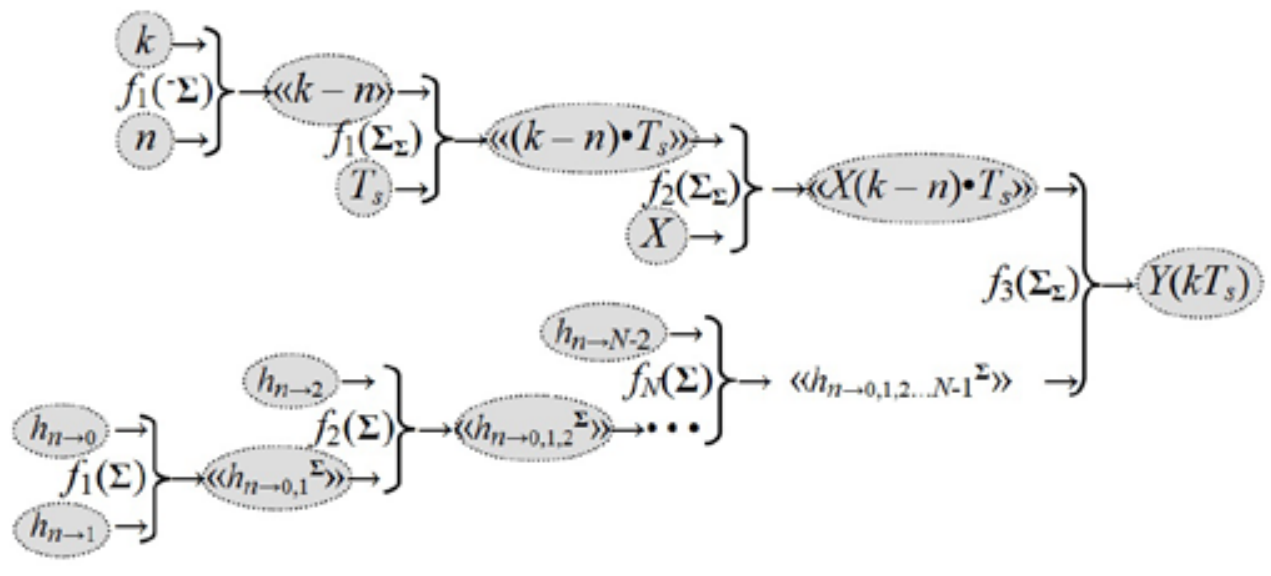

Рис. 7. Графоаналитическая модель структуры цифрового фильтра для вычисления среднего значения периодического сигнала

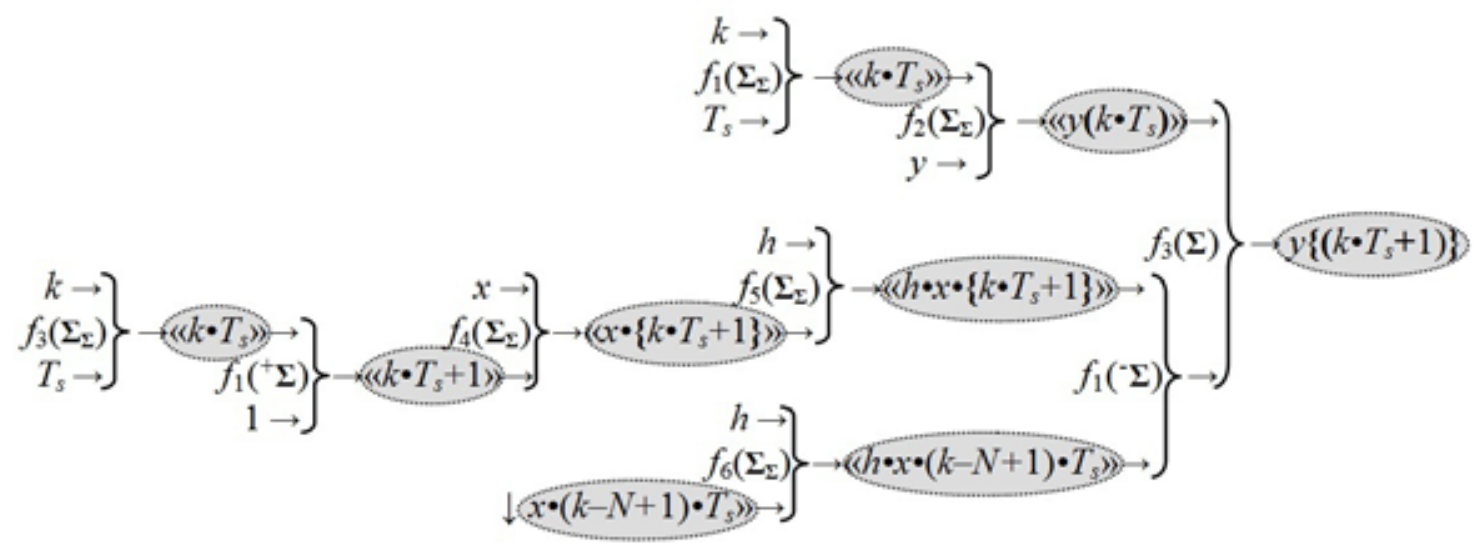

Рис. 8. Графоаналитическая модель оптимизированной структуры цифрового фильтра для вычисления среднего значения периодического сигнала 
пилятора использовался Image Craft ICCAVR Ver $7.22 \mathrm{C}$. Моделирование работы фильтров и определение времени выполнения алгоритмов ЦОС проводилось с использованием AVR Studio 4.12. В результате моделирования было установлено, что эффективность оптимизированной структуры цифрового фильтра растет с увеличением порядка фильтра нелинейно. Для высоких порядков фильтра (более 1000) время вычисления очередного выходного значения цифровым фильтром с оптимизированной структурой приблизительно в 2,5 раза меньше времени, требуемого для аналогичных вычислений цифровым фильтром с прямой (неканонической) реализацией.

Время реакции в рассмотренной структуре цифрового фильтра, реализующего алгоритм расчета среднего и среднеквадратического значений напряжения, составляет $T_{s}$. При этом увеличение частоты дискретизации в оптимизированной структуре фильтра не приводит к возрастанию количества арифметических операций сложения и умножения, что является преимуществом предлагаемой структуры фильтра по сравнению с аналогичными. Если длина линии задержки цифрового фильтра не совпадает с количеством выборок, приходящихся на один период периодических сигналов, в сигнале на выходе фильтра будут наблюдаться колебания. Использование микропроцессорной техники и программной реализации алгоритмов расчета позволяет исключить это явление путем корректировки длины линии задержки цифрового фильтра в зависимости от частоты входного сигнала. Это придает разработанной системе свойство адаптивности, чем не обладают похожие системы.

Выводы. В статье предложен усовершенствованный метод описания структур цифровых фильтров путем формирования графоаналитической формы записи процедуры преобразования информационных аргументов в функциональных структурах. Такой подход дает возможность повысить информационное содержание математических моделей подсистем цифровой обработки сигналов и выполнить анализ системы путем многоуровневой декомпозиции. В свою очередь, это позволяет оценить качество ее структуры и элементов с позиции общего системного подхода, что упрощает проведение интеграционного тестирования системы ЦОС за счет формального определения всех возможных схем взаимодействия аппаратно-программных средств. Практическая ценность полученных результатов состоит в возможности формального решения задачи синтеза оптимизированных структур цифровых фильтров. С использованием предложенного метода выполнена оптимизация структуры системы ЦОС для определения среднего значения напряжения, которую можно использовать в радиоэлектронных и телекоммуникационных системах различного назначения.

\section{Список литературы:}

1. Grout Ian. Digital Systems Design with FPGAs and CPLDs. Oxford : Newnes, 2008. 784 p.

2. Kehtarnavaz N. Digital signal processing system design: LabVIEW-Based Hybrid Programming. Cambridge : Academic Press, 2008. 344 p.

3. Жуков К.Г., Бутусов Д.Н. Реализация цифровых фильтров методом последовательного интегрирования. Научно-технические ведомости Санкт-Петербургского государственного политехнического университета. Информатика. Телекоммуникаиии. Управление. 2009. № 6(91). С. 26-35.

4. Солонина А.И., Клионский Д.М., Меркучева Т.В., Перов С.Н. Цифровая обработка сигналов и Matlab. Санкт-Петербург : БХВ-Петербург, 2013. 512 с.

5. Мефедова Ю.А. Розработка модели для расчета коэффициентов цифрового БИХ-фильтра. Международный научно-исследовательский журнал. 2016. № 4(46). С. 126-128.

6. Campbell St.L., Chancelier J.-P., Nikoukhah R. Modeling and Simulation in Scilab/Scicos with ScicosLab 4.4. New York : Springer, 2006. 330 p.

7. Zhao Jie. Modeling and Simulation of Digital Filter. Proc. 4th National Conference on Electrical, Electronics and Computer Engineering (NCEECE 2015), December 12-13, 2015, Xi'an, China. P. 1333-1338.

8. Kocherzhinskaya Yu.V., Logunova O., Sibileva N. Usage of System Analysis Methods in the Software Products Engineering. Journal of Engineering and Applied Sciences. 2018. Vol. 13. No. 9. P. 3294-3298.

9. Nikolic Sasa, Stancic Goran, Cvetkovic Stevica. Realization of digital filters with complex coefficients. Facta Universitatis, Series: Automatic Control and Robotics. 2018. Vol. 17. No. 1. P. 25-38.

10. Wai-Kai Chen. Passive, Active, and Digital Filter. Boca Raton: CRC Press, 2009. 838 p.

11. Tian Xiao-hua, Zhao Xiao-lei, Zhang Dong, Gao Rui. Design and Implementation of Digital Filter for PMR. Proc. International Conference on Modeling, Analysis, Simulation Technologies and Applications (MASTA 2019), May 26-27, 2019, Hangzhou, China. P. 127-131.

12. Каплун Дмитрий. Цифровые фильтры без умножений. Компоненты и технологии. 2007. № 67. C. $132-135$. 
13. Рябенький В.М., Ушкаренко А.О. Метод синтеза математических моделей логико-динамических процессов контроля и управления. Техническая электродинамика. Тем. выпуск. 2011. № 2. С. 121-125.

14. Kadriu Edona, Bina Lufti. Design of recursive digital filters (IIR). Proc. UBT International Conference, 26-28 October, 2018, Durres, Albania. P. 336-337.

15. Mahmoud M.S. Al-suod, Ushkarenko O.O. Analytical Representation of Control Processes of Induction Motor and Synchronous Generator in Power Plants. Jordan Journal of Electrical Engineering. 2016. Vol. 2. No. 4. P. 278-288.

16. Mahmoud M.S. Al-suod, Ushkarenko O., Petrenko L. Analysis of logical-dynamic conversion processes in arithmetic devices of digital control systems. Eastern European Journal of enterprise technologies. 2017. Vol. 6. No. 4 (90). P. $28-34$.

17. Попов Д. Оптимизация цифровых систем обработки сигналов. Известия высших учебных заведений. Поволюский регион. Технические науки. 2017. № 1(41). С. 96-104.

\section{Ushkarenko A.O. USING OF MULTILEVEL DECOMPOSITION IN THE DEVELOPMENT OF GRAPHO-ANALYTICAL MODELS OF DIGITAL SIGNAL PROCESSING SUBSYSTEMS}

The paper presents an improved method for describing the structures of digital filters, which is featured by the increased information content of the obtained models of digital signal processing systems. The use of the proposed approach allows to synthesize graphic-analytical models of digital signal processing systems and analyze it by means of multilevel decomposition. This makes it possible to assess the quality of its structure and elements from the point of a general system approach and simplifies the integration testing of the digital signal processing system by formal defining all possible interaction schemes for hardware and software. In this case, all elements of the system are presented in the form of functional structures, and the processing of the input sample by a discrete system is considered as a process of converting input arguments using these functional structures. Rules for formal description of elements of digital signal processing systems have been developed and graphical-analytical models of basic structures of FIR and IIR digital filters have been obtained, the distinctive feature of which is increased information content. The practical value of the obtained results is the possibility of using a formal approach to solving the problem of synthesizing optimized structures of digital filters. The proposed method is used in the synthesis of an optimized structure of a digital filter for calculating the average and rms value of a deterministic periodic signal at the optimal speed of the discrete system and invariance to the sampling frequency. The time required to calculate the next output value by the optimized digital filter is 2.5 times less than the time required to perform all calculations when using an unoptimized digital filter structure. The models of digital filter structures presented in the paper can be used in radio-electronic and telecommunication systems for various purposes in the form of embedding units after integration with an object-oriented programming language.

Key words: digital filter, functional structure, analytic model, optimization, argument conversion. 